\title{
RACISMO Y POLÍTICA SOCIAL: NOTAS DE LA MESA REDONDA DEL 6 DE OCTUBRE DE $1999^{1}$
}

Víctor I. García Toro* / Esterla Barreto Cortez**

\section{Introducción}

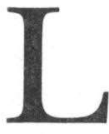

a Mesa Redonda sobre Racismo y Política Social surge como parte de la propuesta desarrollada por el Grupo Puertorriqueño de Estudios de Raza e Identidad de la Universidad de Puerto Rico. En el mismo se integran representantes de diversas unidades académico-investigativos del Recinto de Río Piedras de la Universidad de Puerto Rico, participantes de otros recintos del Sistema de la Universidad de Puerto Rico, de sectores culturales, artísticos, políticos y comunitarios interesados en el tema del racismo. El Prof. Víctor I. García Toro ha sido miembro del referido grupo por un período considerable de tiempo y al surgir esta temática se ofreció de voluntario para desarrollarlo con el apoyo de la Dra. Dagmar Guardiola Ortiz, Coordinadora de la

${ }^{1}$ Celebrado en el Colegio Regional de Carolina de la Universidad de Puerto Rico, bajo el auspicio de la Escuela Graduada de Trabajo Social Beatriz Lassalle y el Grupo Puertorriqueño de Estudios de Raza e Identidad del Recinto de Río Piedras de la Universidad de Puerto Rico. Trabajo presentado por el Dr. Víctor I. García Toro y la Dra. Esterla Barreto Cortez.

* Catedrático, Escuela Graduada de Trabajo Social, Universidad de Puerto Rico.

** Catedrática Auxiliar, Escuela Graduada de Trabajo Social, Universidad de Puerto Rico. 
Propuesta Doctoral entonces e interesada en el tema de la propuesta mesa redonda.

Se inició el proceso de procurar un marco de referencia teórico para enmarcar las discusiones de la mesa redonda y se inició el desarrollo de las preguntas a tales efecto. En el transcurso del mismo y dado a diversos compromisos, la profesora Guardiola Ortiz apoyó la propuesta pero no pudo continuar con ella directamente. Es entonces que se integra al grupo la Dra. Esterla Barreto Cortez y se le da continuidad al proceso de completar el marco teórico y las preguntas de discusión. Se desarrolla entonces una estrategia para llevar a cabo la Mesa Redonda que finalmente se logra realizar el día 6 de octubre de 1999.

\section{Políticas sociales y racismo}

Las políticas sociales impactan, ordenan y en gran medida condicionan la vida de los ciudadanos en las sociedades organizadas, sean estas democráticas o no. Como instrumentos políticos al servicio del Estado, las políticas sociales son herramientas sumamente valiosas como para pasar desapercibidas por los miembros de una sociedad. Por ende, la discusión sobre racismo y política social es de vital importancia y relevancia.

La carta de derechos civiles de Puerto Rico establece que es inconstitucional el discrimen por razón de raza y color. Sin embargo, el alto grado de desigualdad social entre las personas negras y blancas en Puerto Rico resulta evidente en muchos ámbitos de nuestra sociedad. Contraste por ejemplo la proporción de personas negras en las cárceles de nuestro país en contraposición a la falta de representación de personas negras en posiciones de poder en el gobierno estatal. Compare la proporción de negros y negras en las filas del desempleo y las oficinas de beneficios sociales con su proporción en las oficinas de la "Milla de Oro" y entre el sector empresarial. Ante esta realidad es imperativo el estudio de las dimensiones raciales de los procesos de formulación e implantación de las políticas sociales y cómo dichas políticas contribuyen al mantenimiento de las desigualdades raciales en Puerto Rico.

El racismo individual es expresado y ejercido por individuos cuyos prejuicios raciales y en virtud de sus posiciones de relativo poder en el desarrollo e implantación de políticas sociales cometen actos conscientes o inconscientes de discrimen racial. Más allá de 
esta problemática real, existe la dimensión del racismo institucional el cual es mucho más difícil de identificar, entender y enfrentar. Racismo institucional es definido como la imposición a través de las instituciones legalmente sancionadas en una sociedad de una ideología de supremacía de las personas blancas con el propósito de beneficiar y perpetuar la posición de poder y privilegio de las personas blancas como grupo social (Quiñones, 2000; Chisom \& Washington, 1996). Aunque los(as) puertorriqueños(as) somos la mezcla de tres razas, hecho que dificulta hablar de un grupo racial blanco que se beneficia del racismo institucionalizado, no podemos obviar que las instituciones de nuestro país se construyeron hace más de 500 años bajo un esquema racista que se ha mantenido hasta el día de hoy para sistemáticamente excluir a las personas oscuras y negras de nuestro país de acceso igual a los recursos políticos, económicos y sociales mientras es obvio a la vista el control y acceso a estos recursos de las personas más blancas. Por ello no podemos limitar la discusión del racismo a su manifestación más obvia -el discrimen racial-ya que no empece a que el discrimen por razón de raza y color es ilegal y anti-constitucional en Puerto Rico, prevalecen las diferencias raciales y la exclusión de los(as) puertorriqueños(as) negros(as) del disfrute de las oportunidades y privilegios de nuestro país. Como ejemplo podemos pensar en el impacto que la ausencia de un análisis anti-racista tiene en el desarrollo e implantación de políticas sociales mas allá de la evidente problemática de clase en el ámbito de: las escuelas de la comunidad; la política de mano dura contra el crimen; la intervención de la policía en residenciales públicos y barriadas de la zona urbana y el área de Santurce; la falta de familias adoptivas para niños(as) negros(as) en el Departamento de la Familia; el alto porcentaje de confinados negros en las cárceles del país; las políticas de desarrollo económico para las áreas de Piñones y Santurce; la reforma de salud; y las políticas de control de acceso; entre otros. Sin necesidad de investigaciones exhaustivas nos atrevemos a asegurar que cada una de estas políticas sociales y aspectos de nuestra realidad impactan de forma negativa a los sectores más oscuros de nuestra sociedad. Por otro lado, podemos preguntarnos si debe entenderse que el racismo individual que se muestra como patrón dentro de las organizaciones con responsabilidad de ofrecer servicios sociales en Puerto Rico, es sólo posible por la existencia de una estructura y cultura 
organizacional racista que rehúsa reconocer y enfrentar a nivel institucional el problema del racismo.

Además de la dimensión nacional del problema del racismo institucional en la formulación e implantación de políticas sociales existe la dimensión de nuestra relación con los Estados Unidos. La invasión de 1898 fue motivada por intereses de expansión imperialista fundamentados en una ideología de supremacía blanca e impuesta a través del poder militar (García Passalaqua, 1999). Esta relación persiste hasta el día de hoy como lo evidencia nuestra condición colonial y el caso de Vieques. A su vez, esta ha sido reforzada con la imposición de valores y creencias de la cultura blanca norteamericana (racismo cultural) como lo son el individualismo, la competencia y el control personal, entre otros (Reinat, 1998; Nichols, 1998), y el concepto de lo bello como blanco, rubio, esbelto y de ojos claros. Como consecuencia adaptamos políticas sociales federales que son de origen racista (aún cuando la constitución de los Estados Unidos también hace ilegal el discrimen por razón de raza o color) como lo son la reforma de bienestar social, la ley de una falta y estás fuera de los residenciales públicos y la nueva ley de adopción y protección de menores. Cada una de estas políticas sociales ha estado en el debate público estadounidense por su impacto desproporcionalmente negativo en las comunidades afro-americanas y latinas. Es de esperar entonces, aún cuando en Puerto Rico este aspecto no es ampliamente discutido, que estas políticas también impactarán de forma negativa a las personas más oscuras del país y a quienes se mantienen sistemáticamente marginados y excluidos.

Por último, a nivel internacional, no debemos dejar de mencionar el carácter racista de las políticas neoliberales. El neoliberalismo es la globalización de un liberalismo económico con sus nuevas herramientas tecnológicas que, como en 1492, se impone a través del racismo militar y del racismo cultural en las naciones negras, mulatas e indígenas del mundo para el beneficio y el desarrollo de los grupos blancos de poder en naciones blancas europeas y los Estados Unidos. Mientras tanto se mantiene el sub-desarrollo de los países no blancos (Alejandro, 1999) con la ayuda de los sectores de más poder dentro de estos países quienes a su vez son los sectores más blancos del mismo, sino los más occidentados o americanizados (Petras, 2000). Por ende, las políticas neoliberales tienen un impacto racista en el desarrollo e implantación de las nuevas políticas sociales no solo en Puerto Rico sino a través del mundo. Las 
mismas se diseñan para facilitar la apertura de mercados, la privatización y la desreglamentación en favor de los grupos de poder que en último caso son hombres, blancos, dueños de multinacionales y ciudadanos de una de las $\mathrm{G} 7^{2}$.

La falta de diálogo sobre estos asuntos dificulta la comprensión de la naturaleza excluyente de las relaciones raciales. La situación se complica al intentar analizar el racismo en su interacción con factores, tales como clase, género y nacionalidad, dentro de un contexto histórico. Observamos en Puerto Rico una alta correlación entre privilegio económico y color, siendo los grupos económicamente altos predominantemente de tez blanca, mientras que en los sectores pobres hay una mayor representación de personas de piel oscura. Más allá de negar esta realidad y de rehuirle a un diálogo serio sobre la problemática del racismo en nuestro país se hace necesario el desarrollo de un análisis anti-racista. Un análisis que nos permita formular e implantar políticas sociales que verdaderamente promuevan el bienestar y desarrollo integral de todos/as los/as puertorriqueños/ as, independientemente de su condición de raza o color.

\section{Violencia y racismo}

El análisis del racismo como un problema social trae a colación toda una otra serie de variables intervinientes vinculadas a este proceso social de gran preocupación y actualidad. Ciertamente podría pensarse que el racismo es algo del pasado pero abundando en su complejidad y en sus diversas manifestaciones se percata uno como ciudadano y como profesional, que distante de esa realidad su constancia y persistencia continua vigente. Autores en el campo de Trabajo Social como Van Soest (1997), entienden que hay una estrecha relación entre el racismo y la violencia, a la que clasifica como una aflicción global que tiene, a su modo de ver, vínculos con el desarrollo económico, social y humano de la sociedad. De la misma forma estudiosos de la desigualdad social como Gil (1999), entienden que la violencia y sus amplias dimensiones abarcan plenamente el racismo como una de sus manifestaciones mas viles.

Las fuentes de la violencia son diversas y provienen de los propios sistemas sociales y económicos donde se percibe a través de

${ }^{2}$ Las siete grandes potencias son: Estados Unidos, Canadá, Inglaterra, Francia, Alemania, Italia y Japón. 
privaciones y escasez, de falta de acceso a bienes básicos, de la opresión y del propio racismo contra aquellos diferentes en clase, color y extracción socio-cultural. Puede, de la misma forma, la violencia provenir del Estado a través de acciones tales como, tortura física, represión, brutalidad policíaca como la que se observa en las invasiones indiscriminadas de los residenciales públicos en Puerto Rico y la negligencia oficial, entre otras: como la que se observa en los devastadores efectos de los huracanes y otros desastres naturales, sobre las poblaciones pobres y sin vivienda adecuada en este país. De la misma forma para Van Soest, la violencia puede provenir de otros estados a través de guerras, colonización y opresión, como se ha observado contra Cuba a través del embargo o bloqueo existente por más de 30 años por diferencias ideológicas por los Estados Unidos. De la misma forma grupos de individuos pueden violentar los derechos y la vida de otros a través de rechazo étnico, crímenes de odio por razón de preferencias sexuales, por discriminación y racismo, entre otros. A nivel individual, la violencia se manifiesta en homicidios, violencia callejera y asaltos, entre otros.

Se percibe entonces que los focos de atención en las acciones violentas varían contra diversos grupos como lo son: las mujeres, los niños y los envejecientes a través de violencia intrafamiliar, violación física, sexual y ecológica; los niños, a través de abuso físico y la negligencia; poblaciones oprimidas, a través de genocidio, crímenes de odio, racismo y contra uno mismo, a través de acciones cómo el suicidio y las adicciones, entre otras. Es vital verificar y prestar atención a como se define la violencia para no justificarla debido a su intención o motivación por más noble o racional que pueda parecer.. Podría en casos llevar a los individuos a justificar y aceptar diversos tipos de violencia como lo son: el carrasco que ejecuta, conocido en la literatura como el "verdugo afable”, el soldado que pelea en la guerra, el oficial policial, la autodefensa propia, familiar y de la propiedad, que en ocasiones se considera honorable.

Se define violencia como: cualquier acto o situación que hiera la salud y el bienestar de otros, incluyendo ataques directos a la integridad física y psicológica de la persona, como también acciones destructivas que no necesariamente evidencian una relación directa entre la víctima y la institución o persona responsable.

Para entender la violencia como un fenómeno social debe considerarse: 
1. La naturaleza multinivel de la violencia dentro del contexto cultural.

2. los vínculos entre violencia individual, institucional, estructural y cultural y la violencia por omisión, represión y alienación.

Podríamos visualizar el esquema de la violencia como si fuese una pirámide en cuyo ápice está la violencia individual y en cuya base está la cultural y sus valores. Vista así, se compone de acuerdo a Van Soest (1997), de los siguientes niveles:

1. Violencia Individual: son acciones dañinas contra gente o propiedad visible, fácil de discernir, con consecuencias inmediatas, los agresores (y sus motivaciones) y la víctima (y las heridas) son fáciles de identificar y se consideran crímenes castigables. Ej. asesinatos, violaciones, peleas de gangas, balaceras, abuso, violencia familiar (esposa, hijos, marido), rechazo por color de piel y otras. Al estar ubicada en la cúspide de la pirámide se hace visible y reconocible. Esta también se puede entender como violencia hacia sí mismo, como por ejemplo la adicción y el suicidio. Rechazar a una persona por su color o ascendencia o clichés como el consabido "y tu abuela donde está", han sido tradicionalmente una forma abierta de violencia y rechazo, culturalmente aceptada, a pesar de su implicación racista.

2. Violencia Institucional- se refiere a acciones dañinas llevadas a cabo por instituciones que obstruyen de forma evidente y oculta el desarrollo espontáneo del potencial humano; ocurre en burocracias como agencias del gobierno, negocios, prisiones, instituciones mentales, sistemas de bienestar, escuelas, el ejército, por o con frecuencia causadas por políticas consideradas necesarias para control o represión, como por ejemplo: "Mano Dura contra el Crimen" y "Una falta y estás fuera"; que de modo general son sutiles e indirectas y no se consideran crímenes y de así serlo, se justifican como necesarias al bienestar de la comunidad.

Otro ejemplo podría ser, el racismo institucional como una forma de violencia que resulta en :

a. Alto nivel de desempleo de personas negras.

b. Despido de empleados injustificados y bajos salarios de personas negras en comparación a personas de tez clara. 
c. La alta proporción de personas negras en las cárceles del país.

d. Invasiones de comunidades pobres por la Policía del país, donde la mayor parte de los residentes son mestizos, negros y mayormente pobres.

e. Retener ayudas a gentes y comunidades pobres.

f. Negación de servicios y malos tratos a personas negras que procuran ayudas del gobierno. Este tipo de violencia por sus características de modo general es una violencia que no se ve. Se les conoce como micro-agresiones y se dan con diversos grupos socio-económicamente marginados.

g. Altos índices de mortalidad infantil, muertes prematuras y pocas oportunidades de desarrollo.

h. El referirse a las prietas como buenas enfermeras, es una idea basada en tradiciones pasadas de cuido por parte de negras esclavas que no es atributo de raza, sino racismo.

i. La Política del Estado de "Mano Dura Contra el Crimen", que como política incide en comunidades pobres, con alta incidencia de drogas y desempleo. Es como dice Santiago Valle (1994), una sincronización de factores que evidencian racismo por parte del Estado y de sus instituciones.

3. Violencia estructural/cultural. Los valores y creencias que sostienen la violencia institucional y que resultan en acciones discriminatorias y dañinas que resultan de la forma de pensar de una sociedad, valores convencionales, prácticas del día a día, con frecuencia siniestras, difíciles de discernir y de modo general aceptadas como normales.

Ejemplos de Racismo Estructural/Cultural son:

a. La aceptación pasiva del racismo o la negación de éste.

b. Menosprecio y rechazo a las personas negras.

c. La aceptación fácil del joven negro como adicto, delincuente juvenil o criminal.

d. Pensar que las personas negras son buenas para ser atletas y llevar a cabo trabajos rudos.

e. Blanquizar figuras prominentes de nuestro país que eran negros(as) y mulatos(as) como Julia de Burgos, Pedro Albizu 
Campos y Ramón E. Betances. Esta es una preocupación manifestada públicamente por la cantante/política Ruth Fernández.

f. La existencia de una ideología de supremacía blanca que promueve la creencia de que las personas blancas son superiores a las no-blancas.

Para Barndt (1991) esta dimensión ejemplificada por Van Soest se podría visualizar piramidalmente al analizar el racismo como un fenómeno institucional. Para éste, el racismo institucional tiene tres niveles que al igual que la referida autora van desde el racismo individual que se evidencia en la acción racista de un maestro de escuela hacia un niño, que al así hacerlo evidencia que esta acción se apoya de alguna forma en la estructura de la institución. Argumenta el referido autor que en medio de esa pirámide está la acción racista que se evidencia a través de las políticas institucionales que afectan a determinados grupos y que de modo general es indirecta y se manifiesta a través de rechazo, calidad de servicios y productos. Ya a nivel institucional de base, el racismo inclusive evidenciado en una comunidad como institución social se constata a través de aspectos más concretos y difíciles de lidiar que han pasado a ser aceptados pasivamente por la mayoría de la población, por no poder detectarlos.

La opción de Barndt es semejante a lo que trae a colación Santiago Valle (1994) y que recoge de la misma forma lo expuesto por Van Soest, al referirse a que el racismo se evidencia a través de lo que él llama "el fenómeno de sincronización". Al referirse a la sincronización, ese autor trae a discusión el modo peculiar del gobierno lidiar con las crisis provocadas por problemas como el racismo, la pobreza y el desempleo de forma conjunta. Para él, esta sincronización oculta las acciones de corte racista al incluir todas las categorías juntas. Es como marronizar la pobreza, o como ocurre con frecuencia en Puerto Rico, se criminaliza la pobreza, que con frecuencia es más fuerte entre puertorriqueños de la raza negra, donde a su vez los índices de desempleo tienden a ser mayores.

Las bases estructurales y culturales de la violencia donde se encuentra claramente el racismo son difíciles de identificar y aceptar abiertamente, se manifiestan en la aceptación pasiva de la desigualdad y de las necesidades de los otros. De modo general podemos decir que, violencia genera violencia y el discrimen genera a su vez más discrimen. 


\section{Preguntas}

Para reflexionar sobre los asuntos planteados en el marco de referencia se desarrollaron preguntas sobre el impacto del racismo en torno a la formulación e implantación de políticas sociales, los retos que enfrenta el trabajador social y los recursos para desmantelar el mismo:

\section{- Desarrollo:}

¿Cuál es el impacto de la falta de análisis sobre el racismo en el desarrollo de políticas sociales? ¿Cuál es el impacto del desarrollo de políticas sociales que nos tratan a todos- blancos, "trigueños”, mulatos y negros- como iguales, cuando existen factores sociales, políticos, económicos y culturales que nos hacen diferentes, aún cuando somos iguales en dignidad y derechos? ¿Cuáles son las dimensiones raciales de las políticas sociales en Puerto Rico? ¿En qué medida la ausencia de una perspectiva social obstaculiza la lucha contra la pobreza y las desigualdades sociales?

\section{-Implementación:}

¿En qué medida el proceso de implementación de política pública en Puerto Rico se torna racista? ¿Cuáles son los fundamentos estructurales en los cuales se apoya este proceso? ¿Cómo estos procesos promueven la exclusión social de grupos marginados mayormente constituidos por gente negra?

\section{- Retos:}

¿Cuáles son los retos individuales e institucionales en la lucha contra el efecto del racismo en la elaboración e implementación de políticas, leyes, normas y servicios sociales? ¿Cuál debería ser el papel de los trabajadores sociales en la lucha contra el racismo?

\section{- Recursos:}

¿Cuáles son los recursos que tenemos para enfrentar estos retos? ¿Cuál es el rol de la educación y la cultura para enfrentar los desafíos del racismo en la elaboración e implementación de políticas sociales? ¿Qué cambios curriculares deben adoptar las Escuelas de Trabajo Social para producir profesionales comprometidos con la erradicación del racismo? 


\section{Resumen del diálogo}

A. En torno al desarrollo y la formulación de políticas sociales

1. Se planteó que de hecho sí existe un análisis racista en el desarrollo de Políticas Sociales (P.S.) y que precisamente este tiene la función de controlar y preservar el racismo y la desigualdad social. Por ende el racismo es intencional y no surge por casualidad o ignorancia. Por otro lado se planteó que esta acción racista no es siempre una acción consciente de aquellos que desarrollan y formulan P.S. sino que responde a la socialización racista de estos. Se sugirió entonces que lo que se requiere o lo que falta en este proceso de desarrollo de P.S. es de un análisis anti-racista y de una propuesta política para desmantelar el racismo en Puerto Rico. Como ejemplo se señaló que desde la década del 50 el gobierno del ELA ha tomado acciones concretas para impedir el análisis anti-racista. Así mismo somos testigos de una tendencia hacia la blanquización e invisibilización del negro/a en Puerto Rico tanto en el ámbito político donde los/las negros/as y mulatos tenían un rol importante (ejemplo: Betances, Albizu) así como en otros ámbitos sociales. Otro ejemplo presentado fue el uso de la pregunta sobre identidad racial en el Censo 2000. Dicha pregunta no tiene validez en Puerto Rico en la forma en que fue formulado. Sin embargo, se comentó que mantener la pregunta igual a la del Censo 2000 en los EEUU aún cuando pudo haber sido adaptada a nuestra realidad social fue un intento por parte del gobierno de blanquizar al puertorriqueño de manera que seamos aceptados por los EEUU como un "estado de blancos".

2. Se necesita profundizar el análisis/estudio del vínculo entre desigualdad social y racismo, así como entre P.S. y económicas, en especial en el contexto del neo-liberalismo y las consecuencias de esto en el sostenimiento del racismo nacional e internacional donde se utiliza el elemento raza como el "elemento justificador desde donde se tira la raya de la desigualdad social". Los procesos de colonización y neocolonización parten de una base no solo clasista, sino además racista, por ende, las instituciones que se crean a partir de estos procesos preservan el racismo institucional al cual 
responden desde sus inicios. Igualmente importante es el estudio del vínculo entre clase, raza y género, ya que posiblemente el grupo más marginado en nuestro país son las mujeres negras, pobres y con jefatura de familia.

3. Se discutió la necesidad de analizar la problemática del racismo en Puerto Rico con un enfoque que responda a nuestra realidad social y no importando esquemas de los EEUU. Sin embargo, se señaló la necesidad de entender a profundidad el problema y el análisis del racismo en los EEUU. Esto es de vital importancia, ya que por nuestra situación colonial este tiene unas repercusiones serias en la forma en la que se ha venido re-definiendo el racismo en Puerto Rico, especialmente ante las políticas neo-liberales que se espera promuevan aún más la segregación por color y raza en Puerto Rico. Por otro lado la necesidad de entender que el colonialismo en Puerto Rico en relación a EEUU es una manifestación del racismo en este último. Se sugirió por ejemplo analizar las consecuencias racistas de las preguntas de identidad racial y étnica en el censo del 2000 y la situación de Vieques con la Marina de Guerra de los Estados Unidos.

4. Se señaló la falta de estudios, investigaciones y estadísticas sobre el problema del racismo, así como de Trabajadores Sociales preparados en el tema, lo cual dificulta el planteamiento de argumentos anti-racistas a la hora de desarrollar y formular P.S. Se planteó además la necesidad de cuestionar si las P.S. son para el bienestar social o para mantener el racismo en función de preservar la desigualdad social.

5. Se señaló a la familia puertorriqueña como una de las instituciones que mantienen y promueven los valores y la ideología racista.

B. En torno a los retos en la elaboración anti-racista de políticas sociales se identificó:

1. El reclamo de los privilegios de aquellos que se benefician de la construcción racista.

2. La internalización del racismo y los efectos emocionales (el dolor) que causan el enfrentar el racismo, en especial dado la mezcla racial en nuestras familias y la negación de que 
existe este problema. Como lidiar con el sentimiento propio de haber vivido bajo el racismo inclusive dentro de la propia familia.

3. La falta de un análisis nacional anti-racista y por ende de una propuesta política sólida para eliminar el racismo en Puerto Rico.

4. La falta de estudios y estadísticas que describan la extensión del problema y de información necesaria para sostener planteamientos acerca de la problemática del racismo a nivel de la Legislatura de Puerto Rico. Asimismo la falta de estudios sobre la interacción entre los problemas de clase, género y raza.

5. La falta de foros, cursos y otros espacios donde se discuta esta problemática y donde los profesionales en el campo de políticas sociales aprendan cómo enfrentar y erradicar el racismo.

6. La falta de espacios a nivel de las comunidades del país para lidiar con este problema que tanto ha afectado generaciones puertorriqueñas.

C. En torno a los recursos que tenemos actualmente para enfrentar estos retos se señaló la capacidad de los centros docentes en trabajo social en el país y sus facultades para:

1. Llevar a cabo estudios en el tema y prepararnos para deponer en vistas públicas.

2. Utilizar estudios que ya están en desarrollo para añadir la variable raza.

3. Preparar y ofrecer cursos en ésta área.

4. Servir de recursos a organizaciones anti-racista.

5. Recoger testimonios.

6. Llevar información.

7. Servir de agentes de cambio/transformación en las instituciones para las cuales trabajaremos y otros círculos de influencia.

8. Crear espacios para discusión del tema. 


\section{Próximos Planes para darle continuidad a la discusión del tema}

1. La Prof. Luisa Seijo extendió una invitación para llevar a cabo una Mesa Redonda similar a esta en el Recinto Universitario de Mayagüez con la Facultad de Ciencias Sociales. El propósito de la misma sería continuar este diálogo enmarcado en la realidad social de pueblos en el oeste.

2. Se planteó la necesidad de llevar a cabo una segunda mesa redonda para darle seguimiento a ésta. Se sugirió escoger dos terceras partes de las políticas sociales vigentes para hacer un análisis anti-racista de las mismas.

3. Se sugirió enviar copias de este documento a los/las participantes de esta mesa redonda como una forma de darle continuidad al proceso iniciado.

4. Se acordó publicar un artículo al respecto como forma de documentar el proceso iniciado.

\section{Referencias}

Alejandro, R. (1999). Las reformas al sistema de bienestar social en el contexto de la globalización y el neoliberalismo. Ponencia presentada el 30 de abril en la Undécima Conferencia Dorothy D. Bourne en el Anfiteatro \#1 de la Facultad de Educación de la Universidad de Puerto Rico.

Barndt, Joseph (1991). Dismateling Racism. The continuing Challenge of White America. Minnesota: Aysburg, Fortess.

Chisom, R. y Washington, M. (1997). Undoing Racism: A Philosophy for International Social Changes. Lousiana: The People's Institute Press.

Comisión de Derechos Civiles (1998). ¿́Somos Racistas?

Gil, David (1999). "Understanding and overcoming social-structural violence” en _Contemporary Justice Review, V2(1), pp. 23-35.

Hill Collins, Patricia (1998). "The tie that binds! race, gender and US violence in Ethnic and Racial Studies, Vol. 21(5). 
Muñoz Vázquez, Mayra y Idsa E. Alegría Ortega (1995). Discrimen por Razón de raza en los sistemas de seguridad y justicia en Puerto Rico. San Juan, PR: Comisión de Derechos Civiles.

Petras, J. (2000). La izquierda contraataca conflicto de clases en América Latina en la era del neoliberalismo. Madrid: Ediciones Akal.

Quiñonez-Rosado, R. (2000). Integrating Spirituality and Social Action. Monografía sin publicar.

Santiago Valle, K. (1995). "Vigilando, administrando y patrullando a negros y trigueños: del cuerpo del delito al delito de los cuerpo en la crisis del Puerto Rico urbano Contemporáneo" en Bordes, Vol. 2, pp. 28-42.

Van Soest, D. (1997). The Global Crisis of Violence. Washington, D.C.:NASW. 